\title{
The Resurgence of the Tobacco Pipe in the Gulf Region
}

\section{Cother Hajat*}

UAE University, United Arab Emirates

\begin{abstract}
Tobacco use remains the single greatest cause of preventable death in the world. Whilst much has been achieved in tobacco control policy, this has been predominantly to tackle the use of cigarettes by adult males whilst tobacco use has changed direction away from cigarettes towards the new forms of tobacco and in women and children. The Gulf Region has witnessed a rapid rise in the use of powdered tobacco, dokha, smoked in small pipes called midwakh. This article discusses the emerging literature to date which warrants concern for the widespread use, health effects and difficulty in regulation of midwakh. The few studies on usage suggest that over a quarter of students in the United Arab Emirates smoke midwakh regularly. There are initial reports of higher nicotine levels and deleterious health effects compared with cigarette use. Most of the regulatory mechanisms in place, such as bans on smoking in public places and the regulation of sales, are not applicable to the sale of dokha. Whilst the WHO Global Monitoring Framework target of a $30 \%$ relative reduction in tobacco use by 2025 includes all forms of smoking, the vast majority of the current tools at our disposal, from the scant evidence available, are not fit-for-purpose in tackling non-cigarette tobacco use. Further research and shift in tobacco control policy is needed in the Gulf Region and beyond to tackle the burgeoning use of new forms of tobacco such as midwakh.
\end{abstract}

Keywords: Non cigarette tobacco products; Public policy; Surveillance and monitoring; Global health

Tobacco use is the single greatest cause of preventable death in the world today, killing 6 million people every year with a projected increase to 8 million per year by 2030 [1]. Over the 65 years since Richard Doll's studies proved definitively that smoking cigarettes caused lung cancer, the tobacco control movement has grown to counter the clout of the tobacco industry [2]. Many controls have been put in place and measures taken to slow down the progression of cigarette use including 179 countries signing up to the Framework Convention on Tobacco Control (FCTC) Treaty and numerous organizations becoming involved including more recently the Bloomberg and Bill and Melinda Gates Foundations [3-5].

Whilst this activity has been ongoing, targeting predominantly the use of cigarettes by adult males, the tobacco industry has changed direction away from cigarettes towards the stealthy targeting of new forms of tobacco at non-smoking segments of the population, in particular to women and children. New forms of tobacco now include in India, bidis (thin cigarettes wrapped in the tendu or temburni leaf) and pan (chewed betel quid with tobacco), in Indonesia kreteks (clove cigarettes), and in the Middle East waterpipe (also called hookah, shisha, narghile, hubbly-bubbly used to burn a form of tobacco enriched with molasses and other sweet flavours called maasel) and more recently a sharp rise in the use of dokha in the Gulf Region, a form of homegrown powdered tobacco, burnt within one or two inhalations in small pipes called midwakh.

Dokha is a combination of tobacco leaves with a variety of barks, herbs, spices, dried flowers, dried fruit and possibly other, as yet unsubstantiated, substances such as dried ants and shoe polish. Dokha use originated in northern Iran in the 15th century and widespread use among sailors along the Caspian Sea resulted in its introduction into the Middle East in the 1500s [6]. Whilst it was relatively uncommon 10 years ago in the region, the increase in dokha and midwakh use is of particular concern due to the rapidity of its uptake and the potential to appeal to the lesser-tapped child targets. Preliminary studies suggest that a quarter of male secondary school students and university students in the UAE now use midwakh daily [7-9]. To date, the WHO surveys (GSHS, GYTS and GTSS) carried out in the region have not reported on the use of Dokha.
Due to its marketing as a revival of part of the culture of Gulf countries, midwakh appears to be more culturally acceptable than cigarettes. The most common reasons for use of dokha among university students have been reported as a strong sensation of light-headedness, lack of odour, the small dose required to satisfy nicotine cravings, the absence of lip staining, low cost and the view that it was less harmful than other forms of tobacco, attributes that are attractive to children and adolescents with limited access to money and greater concern with their personal appearance and trends [10].

And whilst the use has been predominantly in the Gulf region of the Middle East, numerous North American and European websites have emerged that market midwakh smoking, especially to youth, as the 'the future of tobacco' [11-13]. There are suggestions that midwakh smoking may emerge in Western countries among 'youth sub-cultures' based on the acceleration of websites selling midwakh and dokha and anecdotal observations of sales of midwakh and dokha in specialty stores in Western countries [6,12-16].

As the mean age of onset of midwakh is earlier than of cigarettes, this suggests the possibility of midwakh having a 'gateway effect', introducing the non-smoker to tobacco at an early age and leading to dual tobacco use of midwakh and cigarettes later on, as has been reported for the use of waterpipe $[17,18]$.

Yet there is very little known about the health effects of dokha and midwakh use. There are less than 15 articles in the scientific literature to date but their findings warrant concern. The midwakh is smoked in two inhalations, over a few seconds compared with a few

*Corresponding author: Cother Hajat, UAE University, Public Health Institute, Faculty of Medicine and Health Sciences, I Ain, PO Box 15551 Abu Dhabi, United Arab Emirates, Tel: 00447983201546; E-mail: chajat@uaeu.ac.ae

Received January 13, 2017; Accepted January 17, 2017; Published January 21, 2017

Citation: Hajat C (2017) The Resurgence of the Tobacco Pipe in the Gulf Region Health Care Current Reviews 5: 181. doi: 10.4172/2375-4273.1000181

Copyright: (c) 2017 Hajat C. This is an open-access article distributed under the terms of the Creative Commons Attribution License, which permits unrestricted use, distribution, and reproduction in any medium, provided the original author and source are credited. 
minutes per cigarette. The levels of nicotine, which confer the addictive component of combustible tobacco products, taken in over the course of a few seconds are six times higher than the levels consumed over the course of a few minutes for one cigarette [19]. This 'buzz' or 'dizziness' gives dokha its name in Arabic. Whilst there no studies to date, it is a reasonable assumption that users of midwakh may have higher levels of addiction than cigarette smokers, explaining anecdotal reports of midwakh users waking up in the night to smoke, a phenomenon not widely reported in cigarette smokers. The short term physiological effects of midwakh are similar to those of cigarette use with comparable increases in heart rate, respiratory rate and blood pressure following midwakh use [20]. A series of case-studies suggests that seizures are also a short term consequence of midwakh smoking possibly due to the high level of nicotine intake [21]. The long-term health consequences on cardiovascular and cerebrovascular disease, cancer and lung conditions are also unstudied.

Tobacco control efforts in the Middle East and elsewhere continue to focus almost exclusively on curbing cigarette use (including smoking cessation training of health and education professionals, media campaigns, increased taxation and banning tobacco smoking in public places), despite the alarming new research indicating increased use of non-cigarette forms of tobacco use.

Policies for tobacco control are the mainstay of tobacco cessation efforts but these are readily circumvented by midwakh users. The UAE was amongst the first Gulf countries to enact a smoking in public places ban. Yet midwakh can be smoked in public places with a smoking ban with low chance of detection due to each midwakh lasting for a couple of inhalations and being odourless, such that within a few seconds there remains no or little trace of smoking. These attributes may seemingly suggest that midwakh is benign relative to the lasting odour and visible, lingering smoke produced by cigarette use. The effects of second hand smoke from midwakh use are unknown.

Other tobacco control measures are also hard to implement for dokha and midwakh use. Dokha is grown and sold locally in the UAE and neighbouring countries of Oman and Iran, making the implementation of measures of pricing and taxation very difficult. The price of Dokha is cheaper than cigarettes; a week's supply of Dokha for an average smoker only costs \$3USD compared with \$21 USD for the average cigarette smoker. Even the midwakh pipe may be hired from tobacconist shops for single use at low cost and without the need to purchase the pipe. Dokha is sold loose, by weight and unpackaged, making the measure of messaging and package design also inapplicable. Prevention efforts are also hampered by the inability of the usual smoking detection methods of carbon monoxide breath tests and salivary cotinine tests to differentiate between those who smoke midwakh and those who are exposed to second hand smoke [22]. Additionally, there is as yet little evidence on success rates for quit programme methods for noncigarette tobacco use including midwakh.

So as the tobacco control industry moves ahead to meet the WHO Global Monitoring Framework target of a 30\% relative reduction in tobacco use by the year 2025 , it is conceivable that a new tobacco epidemic may emerge in the form of newer, sleeker, more female and child-friendly forms of tobacco [23]. Whilst the 30\% target includes all forms of smoking, the vast majority of the current tools at our disposal for the prevention, detection and treatment of smoking, were developed for cigarette use and, from the little evidence available, are not fit-forpurpose in tackling non-cigarette tobacco use.

Sixty five years on from knowing that the smoking of tobacco has devastating health consequences, progress in tobacco control moves ahead at a slow pace and maybe requires a change in direction. Traditionally, prevention efforts have focused on tobacco as a whole rather than separately tackling the dual issues of tobacco use of addiction from nicotine and the detrimental health effects of other components such as tar and formaldehyde. This is the approach of some licensed forms of tobacco cessation medication including nicotine replacement therapy in the form of gum and patches, and snus which is a form of nicotine replacement used against the gum and increasingly popular in Sweden and some other countries. Also in this camp are e-cigarettes but with greater contention due to their combustible nature and concern about the effects of second hand nicotine smoke [24].

Another approach seeks to avoid the difficulties in reversing the addiction to nicotine in adult smokers and instead focusing efforts entirely on preventing or restricting the new onset of addiction through initiation of smoking in children. There are currently a couple of countries which already took the bold move of banning tobacco products, including the severe restriction of tobacco sale and use in Bhutan and the complete ban of waterpipe use in Sharjah, UAE. Tasmania and Singapore have taken the lead in the vision of a tobaccofree generation, proposing the adoption of policies to prohibit the sale of tobacco to all persons born after 2000, to ensure tobacco free millennium generations [25]. There remain, however, many obstacles to the success of this initiative such as the supply of tobacco to this cohort by older contacts, the purchase of tobacco by this cohort in nearby territories such as Australia and the continued resistance by the tobacco industry and proponents of the civil liberties argument.

A third movement, and possibly the most likely to succeed, is a call to phase out the sale of tobacco products by 2040 to the entire population [26]. The four priorities proposed in order to achieve this goal are the inclusion of tobacco reduction targets both in the FCTC treaty and also in the post-2015 sustainable development goals. Furthermore, acceleration in FTCT policy implementation in participating countries is called for, together with a UN high-level meeting on tobacco use to galvanise global action towards the 2040 tobacco-free world goal on the basis of new strategies, new resources, and new players [26].

If successful, these approaches could be the long awaited tipping point in the war against tobacco. However, they will only succeed if all forms of tobacco use and not only the readily packaged tobacco sold in the form of cigarettes or other traditional tobacco products are targeted. With the rapid emergence of new means of tobacco use, equally drastic prevention measures seem more necessary than ever. It is time for policymakers and the tobacco control industry to acknowledge the dangers of new forms of tobacco use and step-up efforts to include these in all tobacco control efforts.

\section{Acknowledgement}

This work was conducted in whole by Dr. Hajat. There are no competing interests and no funding was obtained for this study.

\section{References}

1. The Tobacco Atlas, Fourth Edition (2012) Am J Epidemiol 176: 1193.

2. Doll R, Hill AB (1950) Smoking and carcinoma of the lung. BMJ 4682: 739-748

3. http://www.who.int/fctc/WHO_FCTC_summary_January2015.pdf?ua=1

4. Bloomberg Foundation and tobacco control.

5. Bill and Melinda Foundation and tobacco control.

6. Vupputuri S, Hajat C, Al-Houqani M, Osman O, Sreedharan J, et al. (2016) Midwakh/dokha tobacco use in the Middle East: Much to learn. Tob Control 25: $236-241$. 
7. Shemmari NA, Shaikh RB, Sreedharan J (2015) Prevalence of dokha use among secondary school students in Ajman, United Arab Emirates. Asian Pac J Cancer Prev 16:427-30.

8. Jayakumary M, Jayadevan S, Ranade AV (2010) Prevalence and pattern of dokha use among medical and allied health students in Ajman, United Arab Emirates. Asian Pac J Cancer Prev 11:1547-1549.

9. Crookes A, Wolff K (2014) Prevalence of the tobacco product dokha among high school students in Dubai. Subst Use Misuse 49: 1349-1352.

10. Shaikh RB, Vijayaraghavan N, Sulaiman AS, et al (2008). The acute effects of water pipe smoking on the cardiovascular and respiratory systems. J Prev Med Hyg 49: 101-117.

11. http://www.dokhausa.com

12. http://www.facebook.com/pages/Dokha/10312749639377

13. http://www.facebook.com/EnjoyDokha

14. http://www.facebook.com/iconicdokha

15. http://www.facebook.com/medwakh.dokha

16. http://www.facebook.com/midwakh

17. Al-Houqani M, Ali R, Hajat C (2012) Tobacco smoking using Midwakh is an emerging health problem - evidence from a large cross-sectional survey in the United Arab Emirates. PLoS ONE 7: e39189.
18. Soneji S, Sargent JD, Tanski SE, Primack BA (2015) Associations between initial water pipe tobacco smoking and snus use and subsequent cigarette smoking: Results from a longitudinal study of US adolescents and young adults. JAMA Pediatr 169: 129-136.

19. 16th World Conference on Tobacco OR Health. Abu Dhabi United Arab Emirates, p: 103

20. Shaikh RB, Abdul Haque NM, Al Mohsen HAHK, Al Sharbatti S, Mathew E, et al. (2012) Acute effects of dokha smoking on the cardiovascular and respiratory systems among UAE male university students. Asian Pac J Cancer Prev 13 1819-1822.

21. Alsaadi T, Alkaddour AR, Shahrour TM, Shakra M, Turkawi L, et al. (2014) Midwakh-induced seizures: case series from UAE. Epilepsy Behav 39:85-87.

22. 16th World Conference on Tobacco OR Health. Abu Dhabi United Arab Emirates, p: 104.

23. http://www.who.int/nmh/global_monitoring_framework/en/

24. http://health.spectator.co.uk/e-cigarettes-save-lives/

25. www.smokefreetasmania.org

26. Beaglehole R, Bonita R, Yach D, Mackay J, Reddy KS (2015) A tobacco-free world: A call to action to phase out the sale of tobacco products by 2040 . Lancet 385: 1011-1018 